\title{
Muerte súbita por metástasis de melanoma en aurícula derecha. Revisión de los tumores cardiacos
}

\section{Sudden death due to metastasis of melanoma in right atrium. Review of cardiac tumours}

\section{Resumen}

Los tumores cardiacos son entidades clínicas muy raras, sin embargo tienen especial interés desde el punto de vista médico-forense porque pueden ser la causa de una muerte súbita. Presentamos el caso de un varón de 38 años de edad con un melanoma de coroides que fallece de forma súbita cuatro años después de realizarse el diagnóstico. La autopsia médico legal y el estudio histopatológico mostraron un tromboembolismo pulmonar originado sobre una metástasis de melanoma en la aurícula derecha. En el presente artículo llevamos a cabo una revisión general de los tumores cardiacos.

Palabras clave: Tumores cardiacos. Muerte súbita. Metástasis melanoma.

\section{Abstract}

Cardiac tumors are rare clinical entity, however, they have an especial interest in forensic medicine because they can be the source of sudden death. We report a case of a 38-years old male who had been diagnosed with primary choroidal melanoma some years before his sudden death. Forensic autopsy and histopathologic examination revealed pulmonary embolism due to a melanoma metastasis in the right atrium. In addition, in this article we conduct a general review of cardiac tumors.

Key words: Cardiac tumors. Sudden death. Melanoma metastasis.

\section{Introducción}

Los tumores cardiacos son lesiones muy poco frecuentes, pero con interés médico-forense ya que pueden ser una de las causas de muerte súbita.

Por muerte súbita se entiende la muerte natural, no violenta e inesperada que ocurre poco tiempo después de presentarse los primeros síntomas, habitualmente menos de una hora, en un sujeto sin antecedentes clínicos claramente definidos ${ }^{1-3}$. Esta definición varía notablemente según el autor consultado: Di Maio la define como la que ocurre entre segundos (muerte súbita verdadera) y horas (muerte súbita no instantánea) ${ }^{1}$, Finkbeiner la considera como aquella que ocurre en menos de 1 hora $^{4}$, pero también puede definirse (sin darle tanta importancia a la temporalidad de la misma) en base a la forma de presentarse (súbita, inesperada, rápida, imprevista) o al cuadro clínico de base del fallecido (sin patología aguda conocida o en fase de remisión de sus síntomas crónicos) $)^{2,3,5}$

Habitualmente, en los adultos suele producirse como consecuencia de patología cardiovascular, sobre todo enfermedad coronaria aguda, arteriosclerosis, miocardiopatías o valvulopatías. Todas ellas van a ocasionar un cuadro de arritmia cardiaca y llevan a la muerte del sujeto ${ }^{1-3}$.

Sin embargo, es raro encontrar una tumoración cardiaca como causa de la muerte súbita, por lo que el médico forense debe tenerlo en cuenta, ya que la presencia de un tumor a este nivel puede ocasionar un bloqueo del flujo sanguíneo entre las cavidades

\author{
S. Díaz-Ruiz ${ }^{1}$ \\ M. Salguero² \\ J. Jiménez-Martín ${ }^{3}$
}

${ }^{1}$ Médico Forense. Instituto de Medicina Legal de Málaga.

${ }^{2}$ Servicio de Histopatología. Instituto de Toxicología y Ciencias Forenses. Departamento de Sevilla. ${ }^{3}$ Servicio de Anatomía Patológica. Hospital de Antequera.

Correspondencia: Dr. Sebastián Díaz Ruiz Instituto de Medicina Legal Ciudad de la Justicia Fiscal Luis Portero, $\mathrm{s} / \mathrm{n}$ 29010 Málaga

Fecha de recepción: 19.NOV.2010

Fecha de aceptación: 26.ENE.2011 
cardiacas, en las salidas de los grandes vasos o por taponamiento valvular. Los tumores también pueden crecer sobre el sistema de conducción cardiaco, lo que origina una alteración eléctrica con un cuadro de arritmia y un fracaso cardiaco $0^{6,9}$.

Así mismo, también pueden desprenderse pequeños émbolos tumorales de la masa principal intracavitaria y provocar un tromboembolismo masivo. Por último, el tumor puede asentar en la salida de las arterias coronarias o en los senos valvulares, lo que originaría una obstrucción al flujo, provocando una hipoxia miocárdica6-8

La mayoría de los casos de tumores cardiacos se descubren de modo fortuito en necropsias en los que la muerte ha tenido otro origen (incluso violenta) y, por lo tanto, no son atribuibles a esta patología9-11.

\section{Figura 1 \\ Lesión encefálica frontal derecha de aspecto pardo-negruzco.}

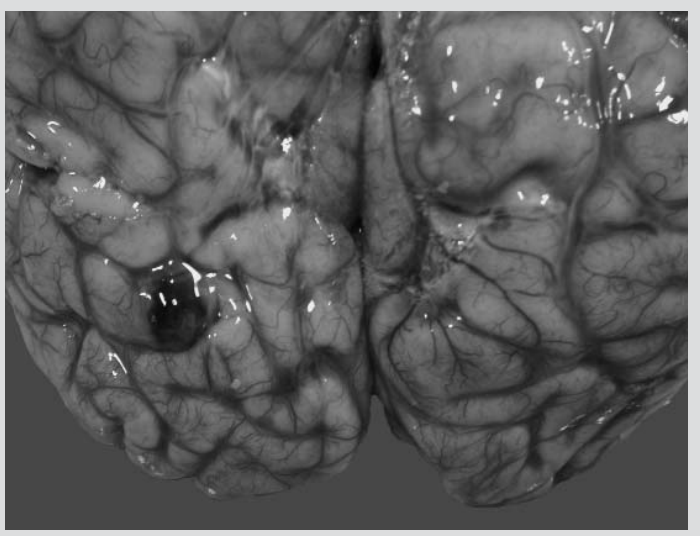

Figura 2. Lesión anterior tras realizar una sección coronal.

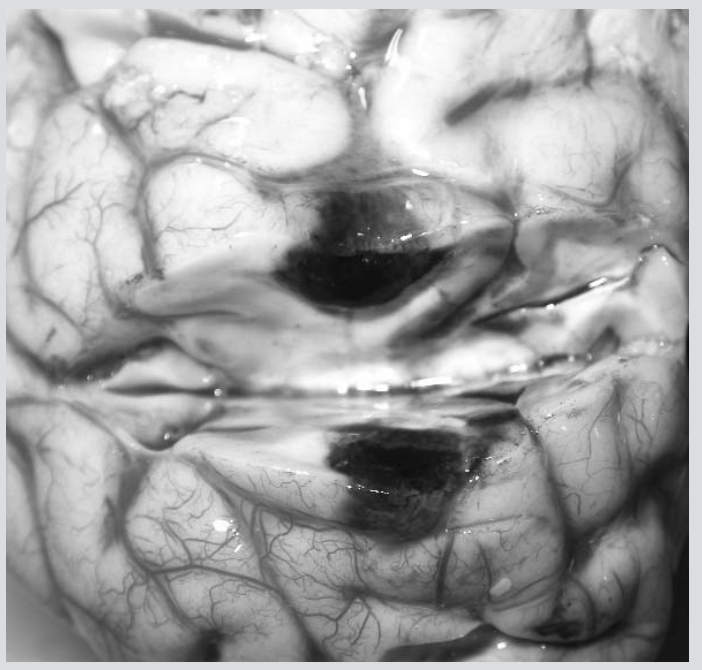

\section{Presentación del caso}

Se trata del cadáver de un varón de 36 años de edad, con antecedente de melanoma de coroides del ojo derecho intervenido quirúrgicamente (enucleación) cuatro años antes del fallecimiento y que, posteriormente, fue intervenido de hepatectomía parcial y cistectomía por posibles metástasis (un año después del diagnóstico inicial del tumor de coroides). Desde entonces, las revisiones médicas, según refiere la familia, han sido normales. Inesperadamente comienza con dificultad respiratoria, falleciendo de forma súbita tras unos minutos, encontrándose junto al cadáver, restos de vómitos biliosos.

En el examen externo destaca tinte ictérico generalizado, restos biliosos que salen por la fosa nasal derecha, edema en ambos miembros inferiores (sobre todo en pie, antepié y tobillo), congestión conjuntival en ojo izquierdo, prótesis de ojo derecho, abdomen globuloso con protusión umbilical, cicatrices quirúrgicas antiguas en región subcostal y flanco abdominal derechos.

En el examen interno apreciamos una nodulación de $1,5 \mathrm{~cm}$ de diámetro en la superficie encefálica frontal derecha, que penetra en la sustancia blanca y de coloración pardo-negruzca (Figuras 1 y 2).

En la cavidad torácica encontramos derrame pleural bilateral y derrame pericárdico de aspecto turbio. En la disección de los vasos pulmonares observamos un trombo de características vitales adherido a la arteria pulmonar derecha que se introduce por las ramas intraparenquimatosas.

En la pared interna de la aurícula derecha existía una tumoración de $1 \times 2 \mathrm{~cm}$ adherida en la pared laterointerna, que ocupaba la cavidad de la orejuela, de coloración pardo-negruzca y aspecto pediculado con amplia base de implantación. La superficie se encontraba discretamente excavada y con una zona de aspecto hemorrágico en el cuadrante infero-interno (Figuras 3 y 4 ).

En la cavidad abdominal existían 1.500 cc de líquido ascítico con restos de heces, adherencias peritoneales marcadas, nódulos linfáticos paravertebrales aumentados de tamaño, de coloración negruzca y duros al corte. Así mismo, también encontramos nódulos con semejantes características adheridos a las paredes peritoneales.

El hígado presentaba una lesión quística con restos de sangre coagulada y material necrótico de coloración verduzca en su interior. La cabeza del páncreas tenía aspecto congestivo y al tacto presentaba consistencia dura y fibrosa. 
Las paredes del intestino delgado aparecían adelgazadas y de aspecto necrótico, con perforaciones en íleon y yeyuno que permitían la salida de material fecaloideo y alimenticio sin digerir. Existían segmentos intestinales de aspecto obstructivo, que coincidían con implantes tumorales necróticos.

El intestino grueso presentaba también áreas de infiltración tumoral de coloración pardo-negruzca (Figuras 5 y 6 ).

Se solicitaron estudios químico-toxicológicos de sangre, humor vítreo y líquido ascítico y estudios histopatológicos de encéfalo, corazón, pulmón y trombos pulmonares, hígado, páncreas, intestino (duodeno, yeyuno e íleon) y ganglios linfáticos paravertebrales y nódulos de la pared del peritoneo.

En los primeros se obtuvieron resultados negativos a todas aquellas sustancias estudiadas.

En los estudios histopatológicos se demostró la existencia de una neoplasia compuesta por células poligonales y fusiformes (Figura 7) dispuestas formando sábanas o nidos sólidos (Figura 8). Estos crecimientos tumorales infiltraban el miocardio auricular pero no se acompañaban de reacción desmoplásica. En el seno de la neoplasia existen zonas de necrosis y en la superficie externa se identifica un trombo adherido a una zona ulcerada. Las células tumorales presentaban nucléolos prominentes y frecuentes figuras de mitosis. Con frecuencia, en el citoplasma existía abundante pigmento melánico.

Los émbolos que se apreciaban en las arterias pulmonares presentaban distintos estadios evolutivos. Unos se encontraban en fase de organización, observándose la presencia de fibroblastos y yemas vasculares que iban infiltrando la formación trombótica (Figuras 9 y 10); otros, en cambio, estaban completamente tapizados de endotelio (Figura 11) y en su seno comenzaban a proliferar los vasos desde la pared arterial; y, por último, los más recientes estaban constituidos por fibrina y ocluían el resto de luz residual (Figura 12) y fueron los responsables del fallecimiento repentino del paciente. La presencia de émbolos en diferentes estadios evolutivos implica que, desde el trombo adherido a la superficie de la metástasis auricular, se han ido desprendiendo numerosos émbolos en un espacio de tiempo que puede oscilar entre 3 y 4 semanas de evolución.

Los hallazgos macroscópicos y microscópicos sustentan el diagnóstico de muerte súbita, secundaria a un tromboembolismo pulmonar masivo, que ha tenido su origen sobre la tumoración maligna metastásica de un melanoma en la cara interna de la aurícula derecha, que se ha ido organizando en distintos estadios

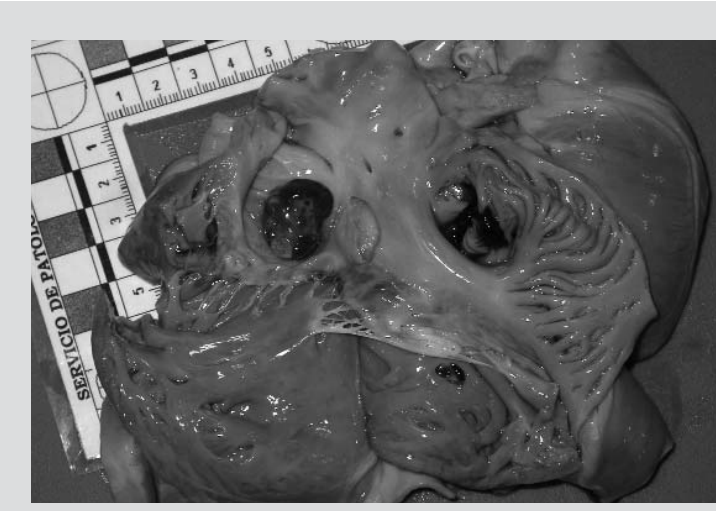

Figura 3.

Lesión nodular

pardo-negruzca en la pared de la aurícula derecha.

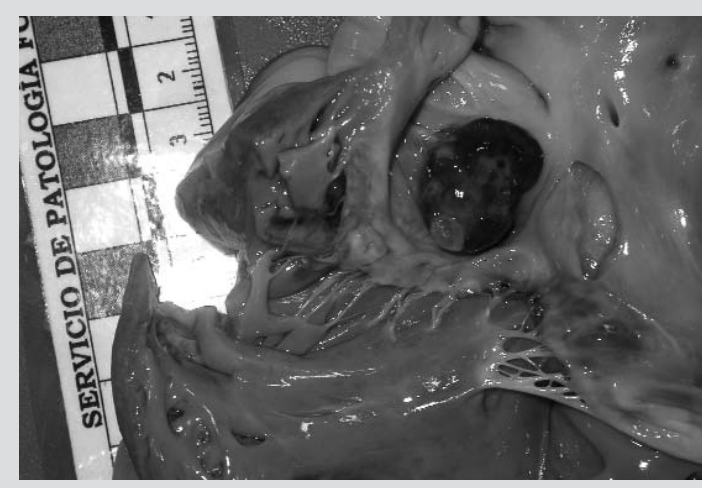

Figura 4.

Detalle de lo anterior,

con zona hemorrágica en el cuadrante ínfero-interno de la misma.

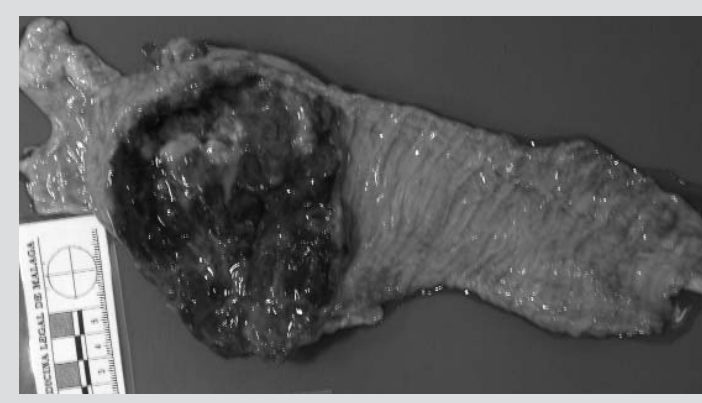

Figura 5.

Lesión infiltrante en mucosa intestinal.

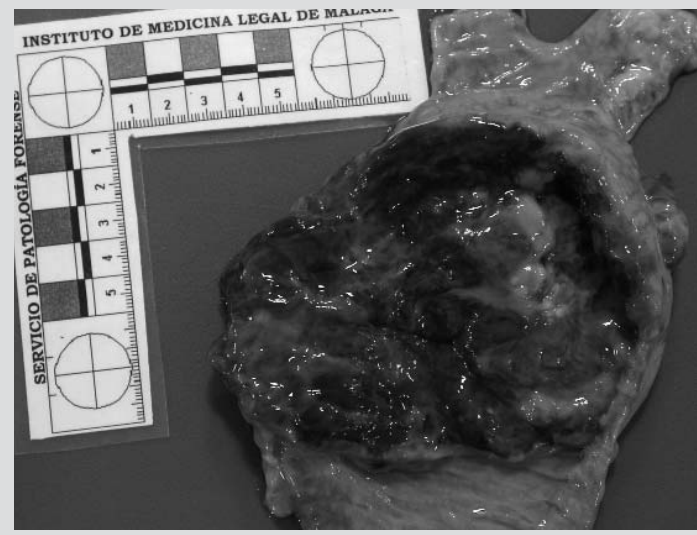

Figura 6.

Detalle de lo anterior. 
Figura 7.

Células con disposición fusiforme y con melanina en su interior.

Figura 8 Células agrupadas en nidos y con pigmento melánico.

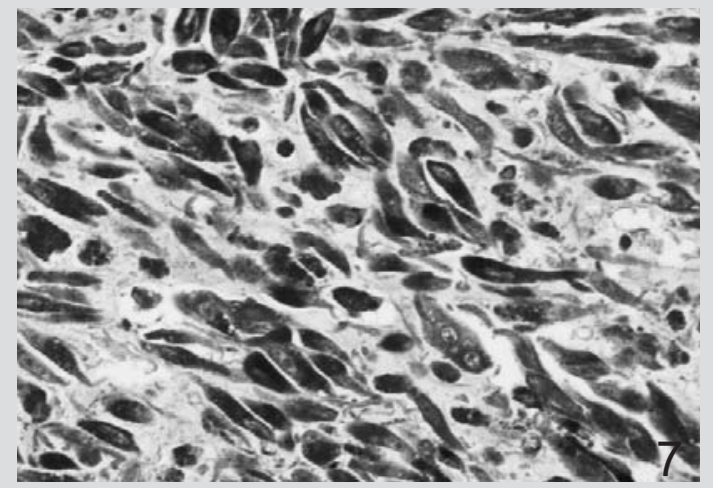

Figura 9.

Pared de arteria teñida con tricrómico de Masson en la que se aprecia una proliferación de tejido fibrovascular que une la pared del vaso con el tromboémbolo.

Figura 10. A mayor aumento se observa que está constituido por fibroblastos y numerosas estructuras vasculares.

Figura 11. Superficie externa de uno de los tromboémbolos en fase de organización tapizada de endotelio.

Figura 12 De izquierda a derecha puede apreciarse la pared vascular, el tromboémbolo reciente constituido por fibrina que ocluye la luz residual y el émbolo más antiguo en fase de organización
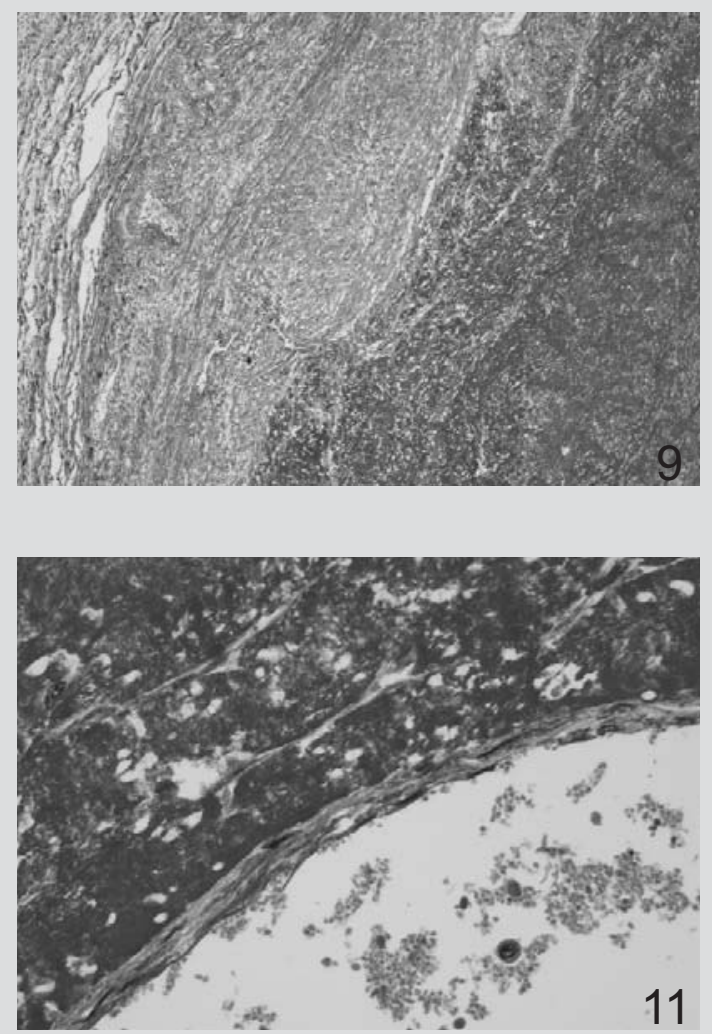

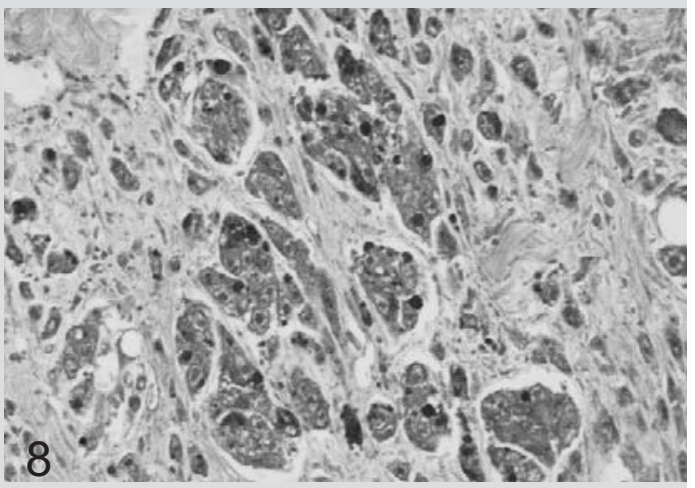

a lo largo de 3 o 4 semanas y que ha finalizado ocluyendo completamente las arterias pulmonares y ocasionando la muerte del sujeto.

Por otro lado, y asociado a lo anterior, nos encontramos con un cuadro de shock séptico por peritonitis secundaria a una perforación intestinal, en el marco de una enfermedad metastásica generalizada cuyo origen es el melanoma de coroides.
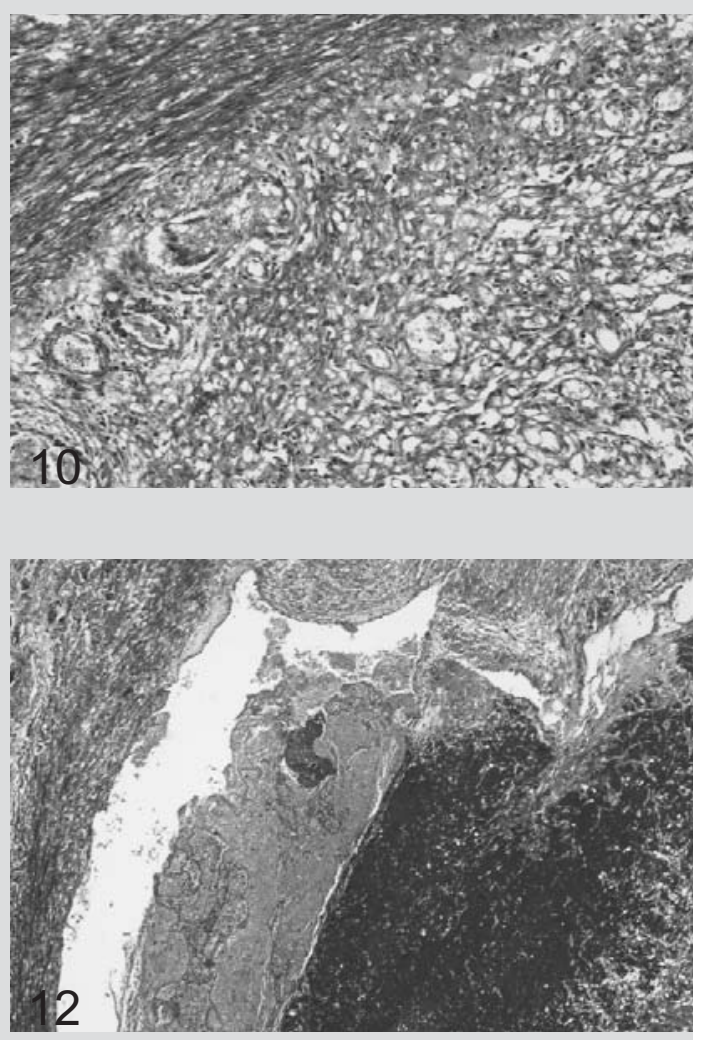

\section{Discusión}

Los tumores cardiacos primarios son lesiones muy poco frecuentes, que según las series estudiadas puede variar desde un 0,001 al 0,03\% de las autopsias generales ${ }^{12}$ y en el $15 \%$ de las autopsias de pacientes con enfermedad carcinomatosa diseminada ${ }^{6}$. El tumor primario más frecuente es el mixoma (75\% 
de los casos) $)^{9}$, pero también pueden encontrarse otras neoplasias benignas tales como: rabdomiomas, fibromas, fibroelastomas papilares, lipomas, hamartomas, teratomas, mesoteliomas y paragangliomas (estos tres últimos tienen una elevada frecuencia de malignización) ${ }^{13}$.

Hasta hace unos años el diagnóstico en vida de estas lesiones era muy poco común pero, en la actualidad, el uso generalizado de la ecocardiografía ha aumentado notablemente su diagnóstico ${ }^{13}$.

Entre los tumores primarios malignos (menos del $25 \%$ de los tumores primarios) destacan los sarcomas (que supone el 95\%) y los linfomas (prácticamente el $5 \%$ restante $)^{14}$.

Los tumores primarios se pueden presentar en todos los grupos de edad, aunque pueden ser un poco más frecuentes en niños y en adolescentes al tener una predisposición familiar, y son más frecuentes en mujeres que en hombres.

Los tumores cardiacos de origen metastásico son entre 100 y 1.000 veces más frecuentes que los primarios, con una incidencia en autopsias del 1,23\%, siendo el carcinoma de pulmón el más común ${ }^{9}$.

El corazón está afectado en 1 de cada 5 casos de tumores con enfermedad generalizada. El $50 \%$ de los melanomas diseminados tiene afectación cardiaca, siendo frecuente encontrarlos en el pericardio visceral y más raramente en el interior de las cavidades (predominan en esta localización las metástasis de sarcomas). La diseminación desde el tumor primario puede producirse por vía hematógena (los carcinomas de riñón, hepatoblastoma y sarcomas de la vena cava inferior habitualmente se localizan dentro de la aurícula derecha), vía linfática (retrógradamente a partir de ganglios linfáticos del mediastino, sobre todo tumores pulmonares, de mama y otros carcinomas) y por continuidad (carcinoma de esófago, pulmón, mama y otros tumores del mediastino), si bien esta última suele ser menos frecuente. No se han descrito casos de metástasis en los que el tumor primario esté en el SNC ${ }^{15}$.

Habitualmente, los tumores cardiacos primarios se localizan sobre todo en el endocardio, seguido del miocardio y el pericardio, mientras que los tumores metastásicos suelen distribuirse de forma inversa, primero en el miocardio (42\%), luego en el epicardio (33\%), pericardio (19\%) y endocardio (6\%). Las metástasis se producen a través de las grandes venas ${ }^{6}$.

El término "corazón melanótico" fue acuñado por WiIliam Norris, en 1850, a propósito de la publicación del primer caso de melanoma metastatizado en el corazón ${ }^{10}$.
La clasificación de los tumores cardiacos puede realizarse en base a diferentes criterios (Tabla 1): origen de la lesión (tumores primarios y tumores metastásicos, y dentro de los tumores primarios se distinguen las lesiones benignas de las malignas) ${ }^{12}$, localización intracardiaca (pericardio, miocardio o endocardio; así por ejemplo, los mesoteliomas suelen asentar de forma preferente sobre el pericardio, mientras que las metástasis de melanoma suelen localizarse de forma casi exclusiva en el miocardio, sin preferencia específica por ninguna cavidad) $)^{6,13}$ y según la frecuencia con que aparece en cada una de las cavidades cardiacas (por ejemplo el mixoma, el osteosarcoma, el leiomiosarcoma y el sarcoma indiferenciado suelen tener preferencia por la aurícula izquierda, mientras que el angioma y el angiosarcoma lo tienen por la aurícula derecha, los fibromas por las cavidades ventriculares, especialmente el ventrículo izquierdo y el tabique interventricular, los rabdomiomas por el ventrículo izquierdo y el fibroelastoma papilar por las válvulas cardiacas) ${ }^{13}$.

\section{Clasificaciones de los tumores cardiacos}

\section{- Según el origen tumoral:}

- Tumores primarios:

* Benignos: mixoma, fibroelastoma, rabdomioma.

* Malignos: osteosarcomas, sarcomas indiferenciados, linfoma de Hodgkin.

- Tumores metastásicos.

- Según la cavidad donde se localicen:

- Aurícula derecha:

* Hemangiomas, angiosarcomas.

- Aurícula izquierda:

* Mixomas, leiomiosarcomas, osteosarcomas.

- Ventrículo izquierdo:

* Fibromas, rabdomioma.

- Ventrículo derecho:

* Metástasis tumorales.

- Válvulas cardiacas:

* Fibroelastomas.

\section{- Según la localización del tumor:}

- Pericardio:

* Mesoteliomas, metástasis de melanomas.

- Miocardio:

* Metástasis tumorales.

- Endocardio:

* Tumores primarios.
Tabla 1.

Distintas clasificaciones de los tumores cardiacos utilizadas en la bibliografía consultada. 
Las metástasis asientan con más frecuencia al corazón derecho (20-30\%) que al izquierdo (10-33\%) y afectan de forma bilateral o difusa en un $30-35 \%$ de los $\operatorname{casos}^{6}$.

Según la primera de las clasificaciones mencionadas $^{12}$, los tumores cardiacos se diferencian en:

- Tumores primarios benignos. Dentro de este grupo destaca, por encima de todos, el mixoma, que generalmente se manifiesta a partir de los 50 años y es más frecuente en mujeres. A veces puede presentarse en el contexto de una enfermedad familiar (complejo de Carnay o complejo "mixoma") que aparece en jóvenes con una pigmentación anormal de la piel (nevus, lentigos), tumores de células de Sertoli-Leydig calcificados, mixomas cutáneos, fibroadenomas mixoides de mama, hiperplasia adrenal cortical pigmentada, hiperactividad hipofisaria, schwanomas y tumores tiroideos ${ }^{12,16}$.

Los mixomas cardiacos son lesiones únicas, pediculadas, móviles y unidas al endocardio. En el $90 \%$ de los casos se localizan en las aurículas, con una proporción de aproximadamente 4:1 a favor de la aurícula izquierda (concretamente sobre la fosa oval que suele ser una de sus localizaciones preferidas), ocupando parte de su cavidad y el $10 \%$ restante en la aurícula derecha. La superficie externa suele ser lisa, fina, gelatinosa y friable al corte, pueden contener zonas quísticas, hemorrágicas, calcificadas e incluso osificaciones. Se pueden llegar a confundir, por su aspecto externo, con trombos murales organizados ${ }^{17}$.

Otro de los tumores de este grupo es el fibroelastoma papilar que suele asentar sobre el aparato valvular y generalmente es infradiagnosticado dadas sus pequeñas dimensiones. La edad media de aparición suele ser los 60 años. Fundamentalmente se localiza en la válvula aórtica y en la mitral, y con menor frecuencia en la tricúspide o en el endocardio. Macroscópicamente tiene forma esférica y está constituido por múltiples papilas tapizadas por células endoteliales hiperplásicas, unidas a un tallo corto compuesto por colágeno y fibras elásticas y musculares lisas ${ }^{16,18}$.

Otros tumores primarios benignos son los rabdomiomas, que son más frecuentes en la infancia y se suelen asociar a esclerosis tuberosa, y característicamente se presentan como masas múltiples casi siempre en las paredes ventriculares ${ }^{17}$, los fibromas (que son los tumores congénitos más frecuentes y que se diagnostican en niños menores de 1 año), hemangiomas, lipomas, la hipertrofia lipomatosa del septo interauricular y los tumores del nodo auriculoventricular, que son extremadamente raros y su importancia radica en su asociación a muerte súbita debido a una arritmia maligna.

- Tumores primarios malignos. Dentro de esta categoría destacan por encima de todos los sarcomas cardiacos, que son los tumores primarios malignos más frecuentes en adultos y se presentan por igual en varones y mujeres. Según el tipo histológico pueden ser angiosarcomas, leiomiosarcomas, rabdomiosarcomas y sarcomas indiferenciados. Los angiosarcomas son las lesiones más frecuentes, suelen ser de alto grado de malignidad y muestran tendencia a metastatizar a pulmón, cerebro, hueso y colon. Macroscópicamente tienen contornos mal definidos, aspecto hemorrágico y asientan preferentemente en la aurícula derecha. Los sarcomas indiferenciados son lesiones lobuladas, de gran tamaño, y a veces pediculadas, que tienen preferencia por la aurícula; histológicamente no presentan signos citológicos de diferenciación específica ${ }^{12,16}$.

Otros tumores primarios malignos son los linfomas cardiacos que suelen presentarse en el contexto de una enfermedad hematológica diseminada. Así, se han descrito hasta en un 20\% de los casos de linfoma de Hodgkin desarrollarán tumores cardiacos a lo largo de la enfermedad ${ }^{13}$.

- Tumores metastásicos. Las metástasis de tumores extracardiacos son las neoplasias malignas más frecuentes del corazón. Actualmente se estima que el $14 \%$ de los tumores resecados son metástasis, siendo más frecuente la afectación pericárdica que intramiocárdica ${ }^{17}$.

Otros tumores que, aunque infrecuentes, van a dar mayoritariamente metástasis cardiacas son los timomas malignos y las neoplasias de células germinales.

Los tumores que son más comunes en la población general suelen metastatizar en el corazón con menor frecuencia, aunque globalmente suponen una cifra elevada dada su prevalencia, como ocurre con los carcinomas gástricos, hepáticos, ováricos y de colon-recto.

- Tumores pericárdicos. Los tumores pericárdicos primarios son lesiones excepcionales y, entre ellos, el $50 \%$ de los casos corresponden a mesoteliomas. Estas lesiones pueden infiltrar todo el pericardio visceral y constreñir el corazón, comprometiendo la función cardiaca, pero es raro que infiltren el miocardio ${ }^{6,12,15}$. Mucho más 
frecuentes son las metástasis de cáncer de pulmón, de mama, del sistema hemato-linfoide o de melanomas.

En cuanto a la presentación clínica, podemos hablar de una triada clásica, si bien no es patognomónica dada la variedad de las entidades que pueden presentarse. Esta triada estaría formada por síntomas y signos de obstrucción intracardiaca (disnea, ortopnea, hipertensión pulmonar, edema pulmonar, síncope o presíncope) ${ }^{19}$, de embolización sistémica (tromboembolismo pulmonar, accidentes cerebro-vasculares, embolias de la arteria retiniana, embolismos en las arterias de las extremidades) y síntomas constitucionales (fiebre, artralgia, pérdida de peso, fatiga) ${ }^{14}$. Estos últimos pueden confundir al médico con múltiples cuadros sindrómicos, como una endocarditis infecciosa, enfermedades del colágeno o cualquier otra enfermedad neoplásica, por lo que hace muy difícil su diagnóstico diferencial.

Pero a veces se presentan síntomas que no se corresponden con esta triada, como por ejemplo alteraciones del sistema de conducción por infiltración tumoral, con cuadros de bloqueos de rama, de preexcitación y bloqueos aurículo-ventriculares ${ }^{6,13}$.

Los tumores cardiacos, hasta hace relativamente poco tiempo, eran hallazgos casuales postmortem, ya que no se diagnosticaban clínicamente porque los electrocardiogramas eran inespecíficos. En la actualidad, el diagnóstico se realiza mediante ecocardiografía ${ }^{10}$. Esta técnica permite la localización de los tumores cardiacos, además de poder apreciar su comportamiento dinámico durante los movimientos cardiacos y, junto al doppler, permite evaluar la presencia o no y el grado de obstrucción del flujo sanguíneo que produce el tumor.

Otras técnicas de imagen utilizadas son la tomografía axial computerizada (TAC) y la resonancia magnética nuclear (RMN); si bien esta segunda es mucho mejor para determinar el tamaño, la localización, la extensión, la infiltración miocárdica del tumor y su vascularización, la primera tiene la ventaja de poder determinar con mayor precisión la caracterización tisular, diferenciando zonas de infiltración grasa o de calcificaciones en el estroma tumoral ${ }^{12,19}$.

El diagnóstico definitivo se lleva a cabo mediante estudio histopatológico. Éste se realiza tras la resección quirúrgica, siempre que ésta sea posible, o bien por biopsia tomada a través de cateterismo guiado por técnicas de imagen, sin embargo esta técnica no está exenta de riesgo ${ }^{10}$.

El pronóstico va a depender de la estirpe celular que los constituyan, pero también es muy importante el grado de crecimiento del mismo y la localización, ya que a pesar de ser un tumor benigno, si su localización y tamaño provocan una obstrucción en la salida de sangre de los grandes vasos o en el paso aurículoventricular, puede llevar a la muerte al sujeto de forma precoz; así mismo, si se localiza sobre el sistema de conducción puede ocasionar una arritmia maligna que lleve al sujeto a la muerte. Por último, las metástasis pueden originar derrames pericárdicos hemorrágicos que lleven a un taponamiento cardiaco y a la muerte del sujeto ${ }^{17}$.

Habitualmente, los tumores benignos tienen buen pronóstico mientras que los tumores malignos presentan un desenlace fatal en poco tiempo ${ }^{12,14}$.

\section{Conclusiones}

La muerte súbita es aquella que ocurre de forma natural, no violenta, inesperada y que sucede poco tiempo después de presentarse los primeros síntomas, habitualmente menos de una hora después de que éstos aparezcan, en un sujeto sin antecedentes claramente definidos. Habitualmente, ésta se produce como consecuencia de una enfermedad coronaria, sin embargo aquí presentamos el caso de una entidad rara, la presencia de un tumor cardiaco metastásico que ha sido el origen del tromboembolismo masivo que lleva a la muerte súbita.

La embolización de material sanguíneo coagulado adherido a la superficie del tumor o incluso de partes del tumor (como en el caso del mixoma) puede provocar un tromboembolismo pulmonar masivo que lleva a la muerte al sujeto o a un tromboembolismo sistémico con afectación preferentemente de los vasos craneales o de las extremidades inferiores.

En el caso que presentamos podemos decir que durante 3 o 4 semanas se ha ido formando un trombo sobre la siembra metastásica y que se han ido desprendiendo pequeños fragmentos que ocluyen progresivamente las luces pulmonares hasta llegar a la oclusión total y la muerte súbita del paciente.

Por tanto, el examen autópsico debe de ser exhaustivo, no centrándonos en las arterias coronarias o en la presencia o no de zonas isquémicas miocárdicas, sino que debemos examinar de forma metódica el corazón para no obviar ninguna lesión, por pequeña que sea, en su interior.

La presencia de un melanoma en un sujeto que asocia cierta patología cardiaca nos obliga a descartar la existencia de una metástasis cardiaca, dada la alta frecuencia de su presentación. 
En la actualidad, y dadas las pruebas de imagen existentes, es difícil que llegue a la sala de autopsia un caso de tumoración cardiaca sin diagnosticar, por lo

\section{Bibliografía.}

1. Di Maio V, Dana SE. Manual de patología forense. Madrid: Ed. Diaz de Santos 2003;37-46.

2. Saukko P, Knight B. Knight's Forensic Pathology. Londres: Hodder Arnold Publishers 2004;492526.

3. Dowling G. Sudden natural death. En: Dolinak D, Matshes E, Lew E. Forensic Pathology. Principles and Practice. Burlington: Elsevier 2005;71-95.

4. Finkbeiner W, Ursell P, Davis R. Autopsy pathology. A manual and atlas. Philadelphia: Saunders Elservier. 2009;131-9.

5. Baroldi G, Fineschi V. Specific heart diseases and sudden death. En: Fineschi V, Baroldi G, Silver MD. Pathology of heart and Sudden death in Forensic Medicine. Boca Raton: CRC press Taylor and Francis 2006;108.

6. Burke A, Virmani R. Tumors of the heart and great vessels. Atlas of tumor pathology. Washington DC: Armed Forces Institute of Pathology. 1996;111-9.

7. Cina SJ, Smialek JE, Burke AP, Virmani R, Hutchins GM. Primary cardiac tumors causing sudden death: a review of the literature. American Journal of Forensic Medicine and Pathology. 1996;17:271-81.

8. Chrissos DN, et al. Multiple cardiac metastases from a malignant melanoma. European Journal of Echocardiography. 2008;9:391-2.

9. Ekmektzoglou KA, Samelis GF, Xanthos T. Heart and tumors: location, metastasis, clinical manifestations, diagnostic approaches and therapeutic considerations. Journal of Cardiovascular Medicine. 2008;9:769-77. que tenemos que recabar todas las pruebas antemortem realizadas para determinar la implicación de dicha tumoración en la causa de la muerte.
10. Gibbs P, Cebon JS, Calafiore P, Robinson WA. Cardiac metastases from malignant melanoma. Cancer. 1999;85:78-84.

11. Basarici I, et al. Obstructive metastatic malignant melanoma of the heart: Imminent pulmonary arterial occlusion caused by right ventricular metastasis with unknown origin of the primary tumor. Heart and lung. 2006;35:351-5.

12. Pujol C, Azcárate P, García M. Tumores cardiacos. Medicine. 2009;10:2919-26.

13. Burke A, Jeudy Jr-J, Virmani R. Cardiac tumors: an update. Heart. 2008;94:117-23.

14. Butany J, et al. Cardiac tumours: diagnosis and management. The Lancet Oncology. 2005;6:219-28.

15. Abad C. Tumores cardiacos (II). Tumores primitivos malignos. Tumores metastásicos. Tumor carcinoide. Revista Española de Cardiología. 1998;51:103-14

16. Basso C, Valente M, Poletti A, Casarotto D, Thiene G. Surgical pathology of primary cardiac and pericardial tumors. European Journal of Cardio-thoracic Surgery. 1997;12:730-8.

17. Schoen FJ. El corazón. En: Kumar V, Abbas AK, Fausto N. Robbins y Cotran. Patología estructural y funcional. Madrid: Elservier Saunders. 2006;618-20.

18. Somers GR, et al. Sudden unesxpected death in infancy and childhood due to undiagnosed neoplasia. An autopsy study. The American Journal of Forensic Medicine and Pathology. 2006;27:64-9.

19. Toro R, Lacal JM, Arana R, Zayas R, Callo G, Piñero C. Síncope en paciente con una masa cardiaca. Anales de Cirugía Cardiaca y Vascular. 2005;11:95-8. 